\title{
The Radiographic and Functional Outcome of Bimalleolar Fractures Treated by Open Reduction and Internal Fixation with Screws/Tbw and Plates: A Prospective Study
}

\author{
Dharmesh Patel*, Avtar Singh, Rajeev Vohra and Sandeep Chauhan \\ Senior Resident, MBBS, DNB (Ortho), University Amandeep Hospital, India
}

*Corresponding author: Dharmesh Patel, Senior Resident, MBBS, DNB (Ortho), University Amandeep Hospital, G T road, Model Town, Amritsar, Punjab, India

\begin{abstract}
Background: Bimalleolar fractures are one of the most common fractures in orthopedic traumatology. As with all intra articular fracture, malleolar fracture necessitate accurate reduction and stable internal fixation. When malleolar fractures are not reduced accurately they may lead to post traumatic painful restriction of motion or osteoarthritis or both. To study the functional and radiological outcome and result of surgical treatment of bimalleolar fractures and to know the complication of open reduction internal fixation in bimalleolar fractures.
\end{abstract}

Materials and Methods: A Prospective review was conducted for 60 patients between January 2018 to December 2019 with closed bimalleolar fracture. Open reduction and internal fixation was done with specific modalities. Patients were evaluated with Subjective and objective assessments of the patients' ankles were done using a modification of the scoring system proposed by Olerud and Molander and radiologically by Kristenson criteria. The functional and radiographic outcome of ORIF and advantages of the procedures were recorded. Functional and radiographic evaluations were performed at immediate post op, 6 weeks, 3 months and 6 months, 1 year after surgery. At each follow up, patients were assessed for syndesmotic reduction, loss of fixation, and implant failure and any arthritis changes. The reduction in quality was evaluated on immediate postoperative radiography.

Results: In the present study of 60 patients with bimalleolar fractures treated by open reduction and internal fixation. Excellent results were achieved in $49(81.7 \%)$ patients, good in $9(15 \%)$, and poor in $2(3.3 \%)$ patient. The patient with poor result had mild pain with activities of daily living, diminution in the abilities to run and to do work, reduced motion of ankle and narrowing of joint space.

Conclusions: Understanding the mechanism of injury is essential for good reduction and internal fixation. The fibular length has to be maintained for lateral stability of the ankle. Anatomical reduction is essential in all intra articular fractures more so if a weight bearing joint like ankle joint is involved. Our series encourage operative intervention within 48 hours in the management of bimalleolar fractures of the ankle as the key for high percentage of good result.

Keywords: Bimalleolar Fracture; Plating; Tension Band Wiring; Olerud And Molander Ankle Score; Open Reduction and Internal Fixation

\section{Introduction}

Ankle fractures are one of the most common lower extremity fractures treated in orthopedics and during the last decades the trend towards surgical treatment has increased [1]. The aim is to restore normal anatomy and biomechanics of the joint [2]. Fractures in general have been identified as a medical Problem throughout history and most of Hippocrates essays (about 300 BC) described

the management of injuries, especially fractures and stated that they were sometimes in connection with the skin. In the treatment he separated between injuries with and without lesions of the skin. In the former, reduction was not performed as the patient would die within seven days [3]. Although Hansmann described the first internal fixation by means of plate and screws in 1858, Arbuthnot Lane developed this method further $[4,5]$. 
The firstCase of surgery in the talo-crural joint was reported by von Volkmann in 1875 [6]. Initially however, surgical techniques of fracture treatment did combine the disadvantages of non-surgical and operative treatment: the osteosynthesis was unstable and the fracture site had been exposed with a high risk of infection and disturbed bone healing [5].

The ankle joint (talocrural joint) consists of three bones; tibia, fibula and talus. There are three articular surfaces: the upper joint surface, which are the dome of the talus and the tibial plafond and the main articulation of the joint; the medial joint surface, between the talus and the inner aspect of the medial malleolus; the lateral joint surface, between the talus and the inner surface of the lateral malleolus of the fibula [6]. Malleolor fracture has varied presentation understanding of the biomechanics of the joint and mechanism of injury is essential for adequate reduction and stable fixation. Ankle fractures are the result of low energy trauma involving twisting injuries. These injuries reflect the relative strength of ligamentous component of ankle mortise compared with bone [7]. Most Ankle fractures are isolated Malleolar fractures accounting for two third patients. Bimalleolar fractures occur in one fourth of the patients and trimalleolar fractures occurring in remaining $5-10 \%$. Open fractures are rare accounting for just $2 \%$ of all fractures [7]. Ankle injuries have great importance as body weight is transmitted through it and locomotion depends on the stability of the joint. Intra articular fractures like bimalleolar fractures need thorough understanding of mechanism of injury, proper anatomical alignment, accurate and stable reduction and fixation with appropriate implants in order to reduce painful restriction of movements and osteoarthritis [8].

\section{Aims and Objectives}

a) To study the functional outcome of surgical treatment of bimalleolar fractures treated with CC screw/TBW and plates.

b) To know the complication of open reduction and internal fixation in bimalleolar fractures.

\section{Materials And Methods}

From January 2018 to December 2019, 60 Bimalleolar fractures fixation were performed using specific modalities implant. The study was conducted at Amandeep Hospital, Amritsar, and Punjab after obtaining the ethical clearance from institutional ethical committee. Initial management was done in the orthopedic emergency area, which included getting standard AP and lateral radiographs of the ankle joint (Figure 1). Distal neurovascular status and clinical signs to exclude compartment syndrome were assessed and documented. Patients with gross ankle dislocation were attempted to be reduced in the emergency itself under sedation after prior consent from the patient and relatives. A below-knee plaster slab was applied to immobilize the joint and analgesics were instituted. The limb was kept elevated to prevent excessive swelling. After routine blood investigations and pre-anesthesia clearance, patients were posted for surgery. An ankle CT scan was conducted in all cases as part of the preoperative planning. Intravenous $1 \mathrm{~g}$ cefazolin was administered 30 min prior to skin incision in the operating room after prior antibiotic sensitivity testing. Patient's age between 18 to 85 years with close bimalleolar fractures were included in this study. Patients with open fractures, active infection at site of injury or other associated fractures in the body elsewhere were excluded. Patients with severe preexisting arthritis in the affected ankle joint, limp, or assisted walk due to some previous or ongoing pathology in the hip or knee joint either in ipsilateral or in contralateral limb were excluded from the study.

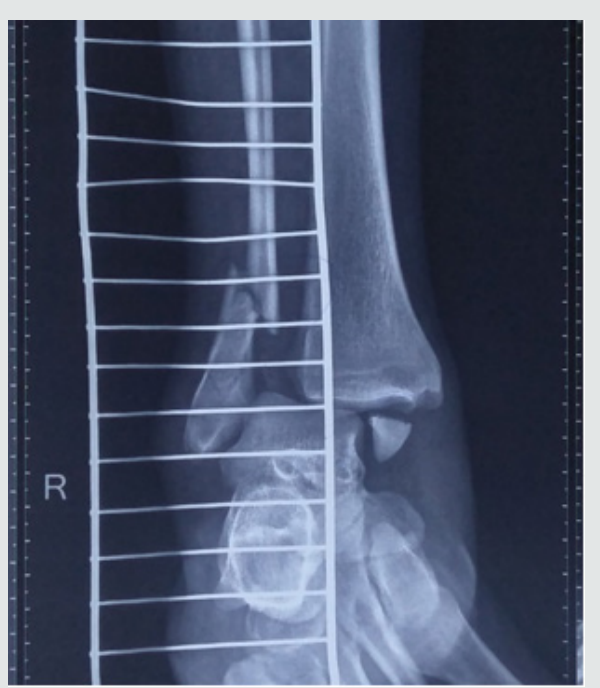

Figure 1: X Ray AP \& Lateral Ankle view showing Bimalleolar Fracture.

Both medial and lateral malleoli were approached by open method. Lateral Malleolus was approached by postero-lateral incision. Adequate reduction done with the help of clamps. Contoured $1 / 3$ rd semi-tubular plate or $3.5 \mathrm{~mm}$ recon plates were used for the fixation. Medial malleolus was approached by $5 \mathrm{~cm} \mathrm{~J}$ shaped incision taken from its tip. Adequate periosteal stripping was done. Reduction achieved with clamps and 2 parallel K wiresanterior and posterior to tip of medial malleolus towards the medial tibial cortex, without entering the joint. Fixed with two $4 \mathrm{~mm}$ CC screws or TBW (Figure 2). The patient was subsequently discharged after a dressing change at $48 \mathrm{hrs}$ post-surgery. A belowknee plaster was maintained until 2 weeks postoperatively till Stitch removal. A strict non weight-bearing and ankle range of motion (ROM) exercises protocol was maintained until 6 weeks post-surgery. Follow-up at 6 weeks was done when radiographs of the ankle joint were repeated and partial weight-bearing with the help of walking aids was initiated. Regular monthly follow-ups were conducted. Full weight bearing was started once the clinical and radiological union was achieved. Ankle score, according to Olerud, et al. [9] (Table 1), and ankle arthritis with weight bearing 
X-rays at 12 months of follow-up were documented in all cases [9, 10]. Post-operative antibiotics were continued for a period ranging from 3 to 5 days depending on the presence of other injuries and therapy was prolonged if there were signs of infection. Once painfree, patient was trained in non-weight bearing crutch walking and advised dorsiflexion and plantar flexion exercises. Postoperative, assessment was done immediately then 6 weeks, 3 months, 6 months and 1 year according to Olerud, et al. [9] functional scoring. Fractures were classified according to Dannis-Weber Classificaton system and operated within $24 \mathrm{hrs}$ of presentation. Subjective and objective assessments of the patient's ankles were done using a modification of the scoring system proposed by Olerud, et al. [9]. Patients were evaluated radiologically by Kristenson criteria [11] (Table 2). Postoperatively complications including Non-union, Delayed union, infection, implant failure, per-implant fracture, and Post traumatic arthritis were recorded.

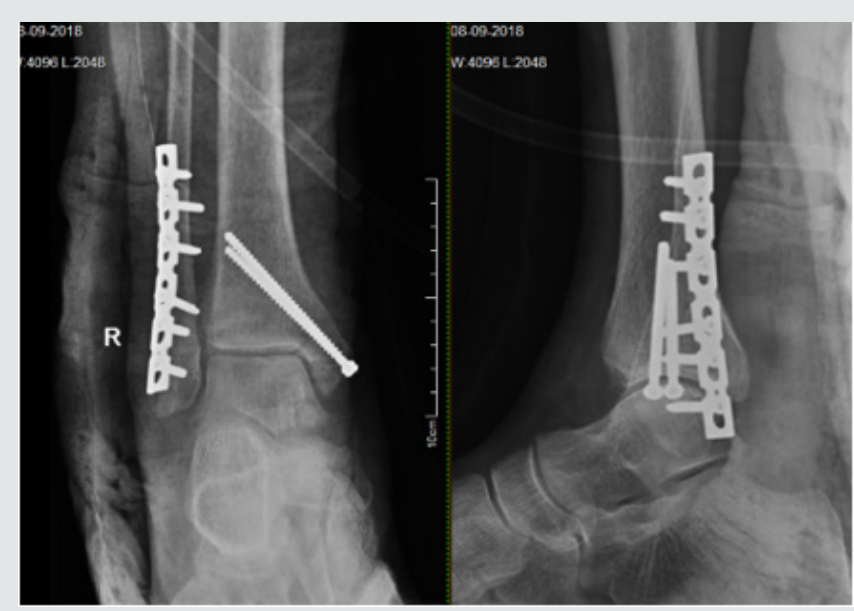

Figure 2: X ray Lt Ankle AP \& lateral showing internal fixation of Bimalleolar fracture.

Table 1: A score of 90 to 100 is considered Excellent; 70 to 89 Good; 50 to 69 points - Fair and less than 50 is considered Poor.

\begin{tabular}{|c|c|c|}
\hline PARAMETER & DEGREt & SCOAE \\
\hline \multirow{5}{*}{ 1. Pain } & None & 25 \\
\hline & While walking on uneven surface & 20 \\
\hline & While walking on even surtace outdoors & 10 \\
\hline & While walking indoors Corstant and severe & 5 \\
\hline & & 0 \\
\hline \multirow{2}{*}{ 2. Stiffness } & None & 10 \\
\hline & Suifness & 0 \\
\hline \multirow{3}{*}{ 3. Swelling } & None & 10 \\
\hline & Only in evenings & 5 \\
\hline & Constant & 0 \\
\hline \multirow[t]{3}{*}{ 4. Stair-dimbing } & No problems & 10 \\
\hline & Impaired & 5 \\
\hline & Impossble & 0 \\
\hline \multirow[t]{2}{*}{ 5. Running } & Possible & 5 \\
\hline & Imponible & 0 \\
\hline \multirow[t]{2}{*}{ 6. Jumping } & Possible & 5 \\
\hline & Impossible & 0 \\
\hline \multirow[t]{2}{*}{ 7. Squatting } & No problems & 5 \\
\hline & Impossible & 0 \\
\hline \multirow[t]{3}{*}{ 8. Supports } & None & 10 \\
\hline & Taping, Wrapping & 5 \\
\hline & Stick or crutch & 0 \\
\hline \multirow{4}{*}{ 9. Work, activities of daily life } & Same as before iniury & 20 \\
\hline & Loss of tempo & 15 \\
\hline & Change to simpler job & 15 \\
\hline & Severely impaired work capacity & 0 \\
\hline
\end{tabular}

Table 2: Kristenson's criteria [11].

\begin{tabular}{|c|c|}
\hline S.N & GOOD \\
\hline 1. & Talus- Correctly Placed \\
\hline 2. & $\begin{array}{l}\text { Medial malleolus - No displacement or fracture gap of less } \\
\text { than } 2 \mathrm{~mm}\end{array}$ \\
\hline 3. & $\begin{array}{l}\text { Lateral malleolus- negligible lateral displacement and up to } \\
\qquad 2 \mathrm{~mm} \text { of posterior displacement }\end{array}$ \\
\hline \multirow[t]{2}{*}{4.} & Posterior malleolus -upward displacement of less than $2 \mathrm{~mm}$ \\
\hline & FAIR \\
\hline 1. & Talus- Correctly Placed \\
\hline 2. & $\begin{array}{c}\text { Medial malleolus - No displacement or fracture gap of less } \\
\text { than } 2 \mathrm{~mm}\end{array}$ \\
\hline 3. & $\begin{array}{l}\text { Lateral malleolus- negligible lateral displacement and up to } \\
\qquad 2 \mathrm{~mm} \text { of posterior displacement }\end{array}$ \\
\hline \multirow[t]{2}{*}{4.} & Posterior malleolus -upward displacement of less than $2 \mathrm{~mm}$ \\
\hline & POOR \\
\hline 1. & Talus- Correctly Placed \\
\hline 2. & $\begin{array}{l}\text { Medial malleolus - No displacement or fracture gap of less } \\
\text { than } 2 \mathrm{~mm}\end{array}$ \\
\hline 3. & $\begin{array}{l}\text { Lateral malleolus- negligible lateral displacement and up to } \\
\qquad 2 \mathrm{~mm} \text { of posterior displacement }\end{array}$ \\
\hline 4. & Posterior malleolus -upward displacement of less than $2 \mathrm{~mm}$ \\
\hline
\end{tabular}

\section{Results}

In our series, most of the patient affected by the fracture belongs to age group of 18- 50 years, which were $42(70 \%)$. The commonest mode of injury is road traffic accident (63.3\%) and fall (36.7\%). 31 were male patients $(51.7 \%)$ and 29 were female patients $(48.3 \%)$. 34 cases involved the right ankle and 26 cases involved the left ankle. The most common injury pattern seen was Dennis Weber Type B in 34 patients (56.7\%). In the present study group, 41 cases $(68.3 \%)$ had a stay of more than 5 days while 19 cases (31.7\%) had a stay of less than or equal to 5 days. The mean duration of stay was 5 days. Surgical technique used was open reduction and internal fixation of the lateral malleolus with semi tubular plate or recon plate; medial malleolus with cancellous screws or tension band wiring. In the present study out of 60 patients, 4 patients presented with persistent swelling, 9 patients presented with residual pain while 12 patients presented with both of the complaints. In our study of 60 cases, 49 cases (81.7\%) achieved excellent results and 9 cases (15\%) achieved good results at 12 months follow up. No significant wound complications were noted. Operative treatment for ankle fractures results in good functional outcome post- operatively. Anatomical reduction of the fracture was associated with better functional and radiological outcomes. Early management with guided weight bearing ensure good functional outcome.

The average time to union and full weight bearing was 12.86 weeks (range 10-16 weeks). The average percentage of the restoration of ROM as compared to the contralateral ankle at the time of union was $90.2 \%$ of dorsiflexion, $91.8 \%$ of plantar flexion, 
$88.1 \%$ of inversion, and $85.1 \%$ of eversion. An excellent outcome in 49 patients and good outcome in 9 patients at the end of 12 months follow-up were concluded according to the Olerud et al. scoring system[9] (Table 1). Bargon criteria[10] for grading post-traumatic arthritis of the ankle joint at the end of 12 months with the help of weight- bearing ankle X-rays were assessed. Only two patients had grade 2 arthritis, eight patients had grade 1 , while the rest had grade 0 arthritis. No complications related to soft tissue healing, pain, or hardware impingement or breakages were encountered.

\section{Discussion}

Understanding the mechanism of injury is important for good reduction and internal fixation. Anatomical alignment and reduction is essential in all intra-articular fractures, more so if a weight bearing joint like ankle joint is involved. More severe injuries showed least satisfactory results and the fibular length has to be mentioned for lateral stability of the ankle. Physiotherapy after cast removal, play a vital role in alleviating post-traumatic ankle stiffness and pain. In the post-operative period, splintage of the ankle and precaution to prevent swelling of the ankle is necessary. The swelling may lead to delayed wound healing. Patients are ambulated with walker without bearing weight on the injured limb from the first post-operative day if there are no associated injuries and can be discharged from the hospital by the second week after removal of suture. Most of the fractures in our study was fixed within 48 hours which however did not change the final outcome. The complications that arose were in those where the fractures were fixed after a week due to swelling which were delayed wound healing and superficial infections of the wound which mostly healed with regular wound care. The six week period of immobilization did not affect the final range of ankle function as most patients had achieved full range of motion by the end of 12 weeks postoperatively with active exercise regimen.

On follow up at 6 weeks, 12 out of 60 patients had persistent swelling and residual pain, 9 patients had only residual pain and 4 patients had only persistent swelling. This is in concordance with a similar study done by Hong et al. [12] in 2014 in which he reported residual pain, swelling and ankle stiffness as the most common complications at 1 year follow up. The mean Olerud et al. [9] score at 3 month post op was 45.91, 6th month post op was 80.33 and at 1 year post op was 94.83. There was a statistically significant improvement in the scores from 3rd month to 6th month post-op (p value 0.001 ). In our study total 53 patients had total score between 90-100, 5 patients had score between $75-89$ and only 2 patient had score less than 75 which is comparable to previous study. Hong et al in 2013 evaluated the functional outcome and limitation of sporting activities after Bimalleolar ankle fractures. At 1 year follow up most patients gained good function and had good to excellent Olreud, et al. [9] scores. However, out of the 47 patients, 26(55.3\%) had residual pain, $29(61.7 \%)$ complained of stiffness and $21(44.7 \%)$ had ankle swelling. Of the 33(70.2\%) patients who were involved in sporting activities prior to the ankle injury, 9(27.3\%) were able to return to pre injury level of sporting activities with no difficulties [13]. According to Kristenson's Radiological criteria out of 60; 49 (81.7\%) patients have good result, $9(15 \%)$ patients have fair result and $2(3.3 \%)$ patient has poor result. Similarly Khandelwal. h et al. [14] in their study recorded Good result in 85\% patients \& Fair result in $15 \%$ patients who were treated operatively.

There are several limitations of our study. The results of this study may be limited by measurement error. The physical measurements may be subject to both, observer's errors and patient variability. Observer's errors can arise from inconsistencies during the recording and reporting of measurements, including; variations in the placement of equipment. The study was conducted by a single observer hence there was no inter - observer bias. Patient variations however, may arise from the patient altering their effort or position when performing the physical assessments, or by reporting a better or worse functional score in response to external influences unrelated to their ankle at the time of completing the score. The study is also limited to patients having surgical fixation for their fracture. These results therefore, cannot necessarily be compared to the outcome achieved with non-operative Management or other modalities of treatment.

\section{Conclusion}

Operative treatment for bimalleolar fractures results in good functional and radiographic outcome postoperatively. Anatomical reduction of the fracture is associated with better functional outcome. Early treatment without delay, anatomic reduction and fracture fixation, stringent postoperative mobilization and rehabilitation should help improve outcome in an operated bimalleolar fracture. Our series encourage operative intervention within 48 hours in the management of Bimalleolar fractures of the ankle as the key for high percentage of good result.

\section{References}

1. Michelson JD (1995) Fractures about the ankle. J Bone Joint Surg Am 77(1):142-152.

2. Ruedi TP, Murphy WM (2000) AO principles of fracture management. Stuttgart, New York: Thieme PP.

3. Lauge N (1948) Fractures of the ankle; analytic historic survey as the basis of new experimental, roentgenologic and clinical investigations. Arch Surg 56(3): 259-317.

4. Lane WA (1909) The operative treatment of fractures. Ann Surg 50(6): 1106-1113.

5. Broos PL, Sermon A (2004) From unstable internal fixation to biological osteosynthesis. A historical overview of operative fracture treatment. ActaChir Belg 104(4): 396-400.

6. Whittle MW, (1996) Gait analysis: An introduction. $3^{\text {rd }}(\mathrm{ed})$. Edinburgh: Butterworth- Heinemann PP.

7. Brown CMC, Heckman JD Rockwood and Green's fracture in adults. Wolterskluwer; $8^{\text {th }}(\mathrm{ed})$.

8. Canale ST, Beaty JH Campbells operative orthopaedics. $12^{\text {th }}$ (ed). Elsevier

9. Olerud C, Molander H (1984) A scoring scale for symptom evaluation after ankle fracture. Arch Orthop Trauma Surg 103(3): 190-194. 
10. Bargon G (1978) Ro“ntgenmorphologische gradeinteilung der posttraumatischen arthrose im oberen sprunggelenk. Hefte Unfallheilkd 133: $28-34$.

11. Joy G, Patzakis MJ, Harvey JP Jr (1974) Precise evaluation of the reduction of severe ankle fractures. J Bone Joint Surg Am 56(5): 979-993.

12. Hong CC, Nashi N, Prasad RS, Ken Jin Tan (2014) Impact of trimalleolar ankle fractures: how do patients fare post operatively? Foot Ankle Surg 20(1): 48-51
13. Hong CC, Roy SP, Nashi N, Tan KJ (2013) Functional outcome and limitation of sporting activities after bimalleolar and trimalleolar ankle fractures. Foot Ankle Int Jun; 34(6): 805-810.

14. Kandelwal H, Rakesh S, Joshi PS, Joshi P (2015) Comparative Study of Conservative and Surgical Treatment of Ankle Fractures. National Journal of Integrated Research in Medicine 6(4).
To Submit Your Article Click Here:

Commons Attribution 4.0 License

DOI: $10.32474 /$ OSMOAJ.2021.05.000204

\begin{tabular}{l}
$\begin{array}{c}\text { Orthopedics and Sports Medicine } \\
\text { Open Access Journal }\end{array}$ \\
Assets of Publishing with us \\
- Global archiving of articles \\
- Immediate, unrestricted online access \\
- Rigorous Peer Review Process \\
- Authors Retain Copyrights \\
\hline $\begin{array}{l}\text { Orthopedics And Sports Medicine: } \\
\text { Open Access Journal }\end{array}$
\end{tabular}

